\title{
Multimodal imaging in a pedigree of X-linked Retinoschisis with a novel RS1 variant
}

\author{
Kirk Stephenson ${ }^{1 *} \mathbb{0}$, Adrian Dockery ${ }^{2 \dagger}$, Niamh Wynne ${ }^{3}$, Matthew Carrigan ${ }^{2}$, Paul Kenna $^{3}$, G. Jane Farrar ${ }^{2}$ and \\ David Keegan ${ }^{1}$
}

\begin{abstract}
Background: To describe the clinical phenotype and genetic cause underlying the disease pathology in a pedigree (affected $n=9$ ) with X-linked retinoschisis (XLRS1) due to a novel RS1 mutation and to assess suitability for novel therapies using multimodal imaging.

Methods: The Irish National Registry for Inherited Retinal Degenerations (Target 5000) is a program including clinical history and examination with multimodal retinal imaging, electrophysiology, visual field testing and genetic analysis. Nine affected patients were identified across 3 generations of an XLRS1 pedigree. DNA sequencing was performed for each patient, one carrier female and one unaffected relative. Pedigree mapping revealed a further 4 affected males.

Results: All affected patients had a history of reduced visual acuity and dyschromatopsia; however, the severity of phenotype varied widely between the nine affected subjects. The stage of disease was classified as previously described. Phenotypic severity was not linearly correlated with age. A novel RS1 (Xp22.2) mutation was detected (NM_000330: c.413C > A) resulting in a p.Thr138Asn substitution. Protein modelling demonstrated a change in higher order protein folding that is likely pathogenic.

Conclusions: This family has a novel gene mutation in RS1 with clinical evidence of XLRS1. A proportion of the older generation has developed end-stage macular atrophy; however, the severity is variable. Confirmation of genotype in the affected grandsons of this pedigree in principle may enable them to avail of upcoming gene therapies, provided there is anatomical evidence (from multimodal imaging) of potentially reversible early stage disease.
\end{abstract}

Keywords: X-linked Retinoschisis, Retinoschisin, Inherited retinal dystrophy, Inherited maculopathy

\section{Background}

Target $5000[1,2]$ (Fighting Blindness, Ireland), the Irish National Inherited Retinal Degeneration Registry, aims to phenotype and genotype all patients in Ireland with inherited retinal degenerations (IRD). A concurrent goal of the study is to facilitate the implementation of individualised management plans including, where appropriate, novel therapeutic options for those patients with modifiable disease. This paper is focused on the

\footnotetext{
* Correspondence: kirkstephenson@hotmail.com

${ }^{\dagger}$ Kirk Stephenson and Adrian Dockery contributed equally to this work.

${ }^{1}$ The Catherine McAuley Centre, Mater Private Hospital, Nelson Street, Dublin

7, Ireland

Full list of author information is available at the end of the article
}

application of this process for a pedigree with X-Linked Retinoschisis (OMIM: 312700, XLRS1) and the clinical and genetic workup of these patients for potential new therapies and future participation in appropriate trials now emerging for this form of IRD.

XLRS1 is a rare IRD (1:15000-30,000 [3]) due to variants in the $R S 1$ gene encoding the retinoschisin protein (OMIM: 300839, Xp22.1). XLRS1 is a mutationally heterogeneous disorder with over 230 known variants [4-6], the majority of mutations falling in exons 4-6 (the structurally important discoidin domain of the retinoschisin protein [7]). Congenital poor vision in males is due to macular schisis and results in variable outer retinal atrophy with age. As novel gene therapies are in clinical trials $[8,9]$, to

(c) The Author(s). 2018 Open Access This article is distributed under the terms of the Creative Commons Attribution 4.0 International License (http://creativecommons.org/licenses/by/4.0/), which permits unrestricted use, distribution, and reproduction in any medium, provided you give appropriate credit to the original author(s) and the source, provide a link to the Creative Commons license, and indicate if changes were made. The Creative Commons Public Domain Dedication waiver (http://creativecommons.org/publicdomain/zero/1.0/) applies to the data made available in this article, unless otherwise stated. 
optimise options for patients, confirmation of the presence of an RS1 variant is essential and moreover must be supplemented with various clinical assays to assess the stage of disease and potential suitability for treatment.

With wide variability in phenotype between and within families [10-12], clinical diagnosis can be challenging. Multimodal imaging has been an excellent addition for confirming phenotype and guiding molecular genetic testing. Optical Coherence Tomography (OCT) imaging may detect intraretinal cystic spaces [13], which may appear in multiple retinal planes. Fundus autofluorescence (AF) imaging can detect subtle patterns of change in the retinal pigment epithelium (RPE), which allows staging of severity [14]. Microperimetry can delineate areas of residual function [15]. Thus, multimodal imaging can be used to demonstrate maintenance of anatomy which may determine suitability for novel treatments.

The RS1 genomic location was first implicated as pathogenic using multipoint linkage analysis of Dutch families affected by XLRS1 [16]. Locus localization was later refined and the RS1 gene was identified by positional cloning. Mapping and expression analysis led to the discovery of the then novel transcript (XLRS1) [17] encoding the retinoschisin protein.

Retinoschisin, a 224 amino acid protein, is secreted from photoreceptors and bipolar cells in retina and has been shown to be crucial for cell adhesion during retinal development [18]. It has been suggested that retinoschisin may also be involved in mitogen-activated protein kinase signaling and apoptosis in retina [18] and recently has been shown to influence $\mathrm{Na} / \mathrm{K}$-ATPase signaling and localization [19]. Employing cryo-electron microscopy, it has been discovered that functional retinoschisin forms a dimer of octamer rings comprising a hexadecamer. Given this intricate structure, a mutation that affects the folded structure of the RS1 protein monomer is likely to inhibit the proper assembly of the 16-subunit oligomer $[20,21]$ as evidenced by the large number of reported pathogenic mutations [5]. Pathogenic RS1 mutations are typically associated with XLRS1, although some mutations show greater phenotypic variability than others, including greater ranges of onset age and severity of condition [12].

\section{Methods}

\section{Clinical phenotyping}

A large pedigree with a clinical diagnosis of XLRS1 was invited to participate given informed consent. Nine affected males ( 7 adults, 2 children), one carrier female and one unaffected male attended the recruiting hospitals as specified above to take part in the IRD registry (Fig. 1).

Phenotyping included an ophthalmic and medical history, pedigree mapping, and dilated ophthalmic examination. Colour photography, autofluorescence (AF, Optos plc, Scotland) and spectral domain optical coherence tomography (SD-OCT, Cirrus HD-OCT, Carl Zeiss Meditec AG, CA, USA) images were acquired. This multimodal imaging was assessed and the macular findings were categorised into their appropriate stages. Stage was assessed by individual eye, not entire patient.

\section{DNA isolation and next generation sequencing}

Blood samples were taken from patients after clinical assessment. DNA was isolated from $2 \mathrm{ml}$ of blood and fragmented for targeted sequencing to an average fragment size of 200-250 base pairs. Sequencing libraries were generated and target capture was performed with the Nimblegen SeqCap EZ kit (Roche), incorporating the exonic regions of over 200 genes implicated in IRDs. Capture regions also included intronic regions in the CEP290, ABCA4 and USH2A genes that are known to potentially contain pathogenic mutations [22-24]. The total size of the captured region was approximately $750 \mathrm{~kb}$.

Captured patient DNA was multiplexed into 24-sample pools and sequenced using an Illumina MiSeq. Confirmatory single-read sequencing was also performed to verify the presence of candidate mutations.

\section{Polymerase chain reaction and sanger sequencing}

To validate the $R S 1$ variant identified by NGS, an amplicon for direct Sanger sequencing was designed incorporating the variant. The sequence used for primer design was Human reference transcript NM_000330.3. Forward primer: 5' - GCAGATGATCCACTGTGCTG - 3'. Reverse primer: 5' - TTTCTTGGGAGGTGGAGATG - 3'. Oligonucleotides were purchased from Sigma-Aldrich (www.sigmaaldrich.com/). The target DNA products were

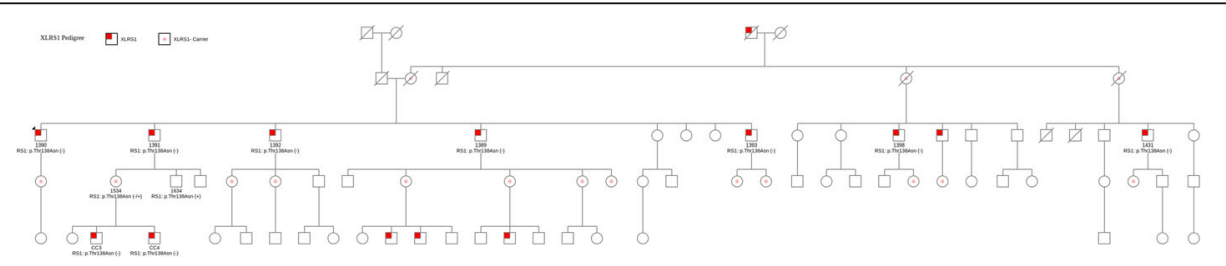

Fig. 1 Pedigree of 5 generations of an XLRS1 pedigree. Individuals marked with a red square were confirmed to be clinically affected. RS1 genotype has been annotated within the pedigree tree for those investigated 
amplified using Q5 High-Fidelity 2x Master Mix (New England Biolabs Inc). The annealing temperature for reactions was $65^{\circ} \mathrm{C}$; all other details were executed as per the supplier's recommendations. PCR products were purified using the GeneJET Gel Extraction Kit (Thermo Fisher Scientific). Sanger sequencing was performed by Eurofins Genomics (www.eurofinsgenomics.eu).

\section{Data analysis}

Data obtained from NGS was subsequently demultiplexed and mapped to the human genome (hg38) using BWA version 0.7.15 [25]. Duplicate reads were flagged using Picard version 2.5.0 [26] and downstream analysis and variant calling performed using Freebayes version 1.1.0 [27].

Variants were identified and scored based on methods outlined in Carrigan et al. [1]. Synonymous variants, polymorphisms and mutations with high frequency in any population were filtered out, and the remaining list of rare variants with the potential to affect protein sequence was output for manual inspection. Output scores from the following ensemble variant pathogenicity predictor tools are shown in Table 1. The scale for each score ranges from 0 (likely benign) to 1 (likely pathogenic).

\section{MetaLR}

Meta Logistic Regression (LR) [28] incorporates pathogenicity prediction scores and maximum minor allele frequency from nine different tools for more accurate and thorough evaluation of deleterious effect of missense mutations. This allows for the more accurate assessment of variants than any of the singular methods alone.

\section{$M-C A P$}

Mendelian Clinically Applicable Pathogenicity (M-CAP) Score [29] was the first high sensitivity pathogenicity classifier for rare missense variants in the human genome aimed at the clinic. It combines pathogenicity scores from other tools and databases (including SIFT, Polyphen-2 and CADD) with novel features to create a more powerful model.

\section{Revel}

REVEL (rare exome variant ensemble learner) [30], is also an ensemble predictor tool and was trained with recently discovered pathogenic and rare benign missense variants, excluding those previously used to train its constituent tools. REVEL also claims to have the best performance for categorising pathogenic from rare benign variants with allele frequencies $<0.5 \%$.

The American College of Medical Genetics and Genomics (ACMG) criteria for classifying pathogenic variants was utilised [31]. The other rare variants detected were for autosomal recessive conditions and showed pathogenic variants in only 1 allele. This was consistent between affected male relatives in this study.

\section{Protein modelling}

3D models of single subunit wildtype RS1 (NM_000330.3) and the Thr138Asn mutant were generated using Iterative Threading ASSEmbly Refinement, I-TASSER [32]. Polymer structures of RS1 were obtained from the Protein Data Bank (PDB, ID\#3JD6,) [33]. The effect of single point mutations on protein stability was measured using STRUM [34]. Protein alignments were generated using Clustal Omega [35].

\section{Results}

Mean patient age of affected adult males $(n=7)$ was 67 $+/-5.38$ years. The recruited carrier female (daughter) was 47 years of age. Mean age of the 2 affected grandsons was $13+/-1.41$ years. Mean visual acuity for adult affected males was LogMAR $0.78+/-0.16$ for right eyes and $0.74+/-0.28$ for left eyes. Two eyes were not assessable due to one case of dense cataract (amblyopic eye) and one enucleation (due to complications following previous retinal surgery, presumed post vitreous haemorrhage or detachment). Only one of 14 adult eyes developed either a retinal detachment or haemorrhage previously (indeterminable). Mean visual acuity of affected grandsons was LogMAR $0.6+/-0.14$ for right eyes and 0.5 for both left eyes.

Macular changes varied in severity, which showed no correlation to patient age. A severity staging was proposed by Tsui and Tsang [14], outlined in Table 2. Of 12 assessable adult eyes, 1 was in Stage 1 (8\%, mean age $74 y), 6$ were in Stage $2(50 \%$, mean age $65.6+/-5.72 y)$ and 5 were in Stage 3 (42\%, mean age $68.8+/-4.55 y)$. The predominantly advanced stage of disease here is likely due to advanced patient age. Corresponding images from this series are in accordance with this and are shown in Fig. 2. Both affected grandsons had bilateral Stage 1 disease. Electrophysiology data for the 2 affected grandsons is shown in Fig. 3. Confirmation of this RS1 variant's pathogenicity will

Table 1 A direct comparison of the novel mutation, p.Thr138Asn, and a known pathogenic variant in RS1. Both variants were analysed with the same bioinformatic pipeline and have been scored by the same computational tools

\begin{tabular}{llllllll}
\hline Variant & Nucleotide & Protein & DBSNP ID & MetaLR & M-CAP & REVEL & ClinVar Report \\
\hline Novel & c.413C $>$ A & p.Thr138Asn & None & 0.9742 & 0.8775 & 0.929 & None \\
Known & c.636G $>$ A & p.Arg209His & rs281865362 & 0.9446 & 0.9233 & 0.796 & Pathogenic \\
\hline
\end{tabular}


Table 2 The clinical staging of Retinoschisis [14]

\begin{tabular}{llll}
\hline Stage & Colour & AF & SD-OCT \\
\hline 1 & Macular schisis, classically & Macular pigments displaced between schitic areas (B) & $\geq 1$ intraretinal schisis plane (C) \\
2 & radial (A) & Hyperautofluorescent ring at macula (E) & Collapse of schisis plane(s) (F) \\
3 & Macular atrophy (G) & $\begin{array}{l}\text { Large area of central hypoautofluorescence } \\
\text { with surrounding hyperautofluorescent ring (H) }\end{array}$ & Outer retinal atrophy (I) \\
\hline
\end{tabular}

be of most benefit for this younger generation with potentially reversible macular changes.

Figure 2 demonstrates the clinical staging of XLRS using multimodal imaging. Patient 1 (74y) demonstrates the features of Stage 1 (mild) disease with BCVA LogMAR 0.6. There are subtle pigmentary changes on colour photography (A), with displacement of macular pigment leading to radial pattern of hyper- vs hypo-autofluorescence (B). The most striking feature is on OCT with intraretinal cystic spaces (C) in the inner plexiform and inner nuclear layers.

Patient 2 (60y) shows Stage 2 (intermediate) disease with BCVA of LogMAR 0.9. Colour photography reveals a hypopigmented ring at the macula, surrounding an area of normal pigmentation (D). Autofluorescence confirms this as hypoautofluorescent with a surrounding ring of hyperautofluorescence and a preserved iso-autofluorescent foveal area (E). OCT reveals the absence/collapse of intraretinal cystic spaces, but lacking complete outer retinal atrophy (F). Incidental vitreo-foveal traction is noted in this case.

Patient 3 (71y) has stage 3 (advanced) disease with BCVA of LogMAR 1.0. The colour photograph demonstrates macular atrophy (G), which appears as hypoautofluorescence with a hyperautofluorescent ring $(\mathrm{H})$. This corresponds to outer retinal atrophy without schitic intraretinal space on OCT (I).

Next generation sequencing detected a novel missense mutation in RS1 (c.413C > A; p.Thr138Asn [1]) falling within exon 5 of the discoidin domain [7]. This is a variant of unknown significance; however, due to its location adjacent to known pathogenic loci, it was deemed to be

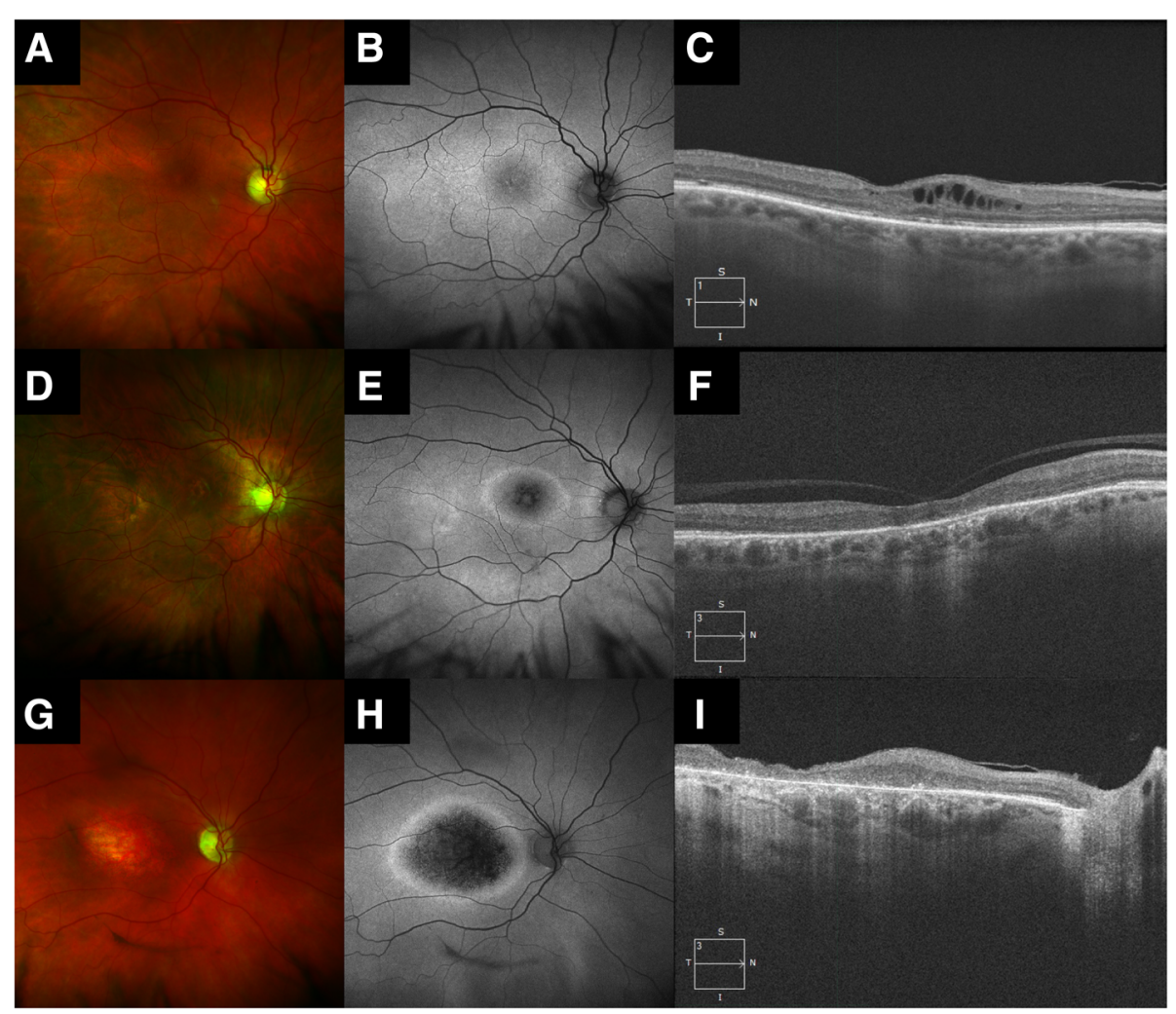

Fig. 2 Phenotypic features of selected cases from this XLRS1 pedigree. Colour photographs (left column), autofluorescence (middle column), and SD-OCT (right column) of the right eyes of 3 patients in Stage 1 (top row), Stage 2 (middle row) and Stage 3 (bottom row). Further description is available in Table 2 and the 'Results' section 


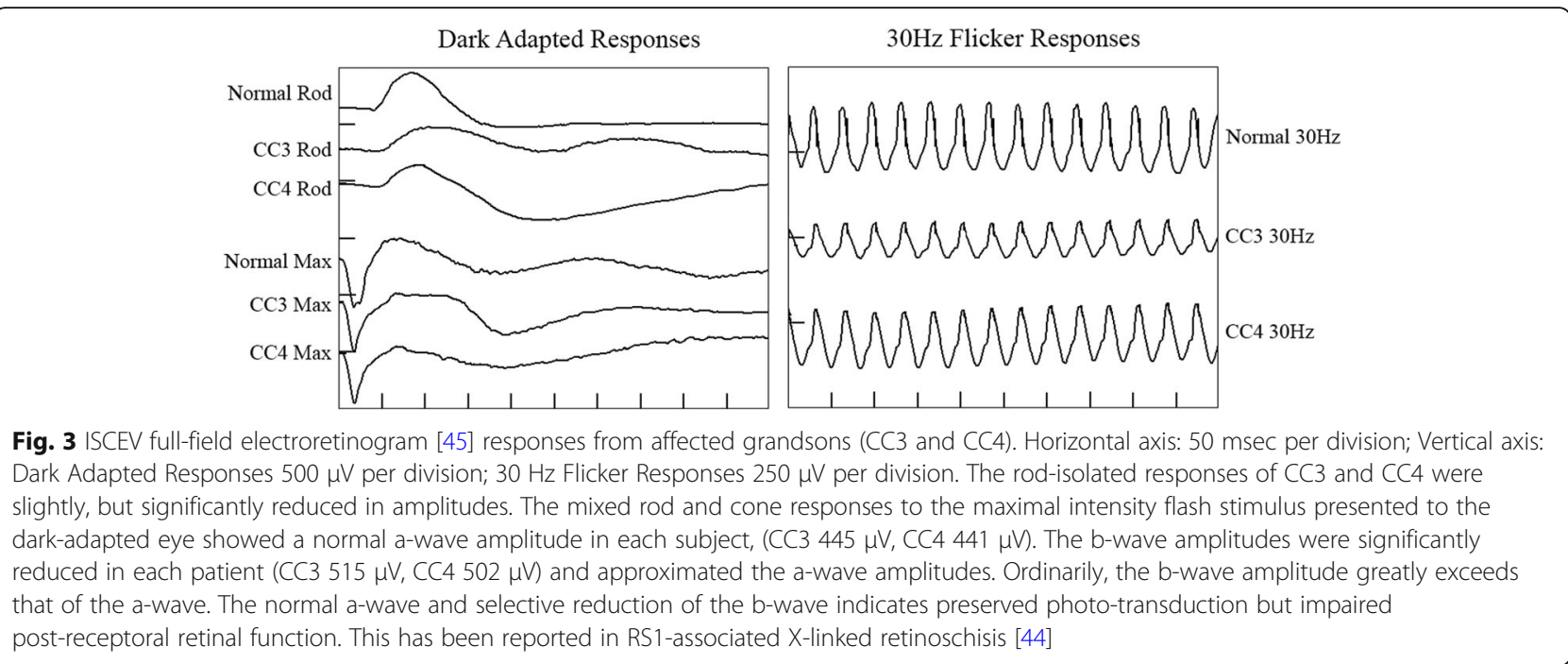

potentially pathogenic and in silico analysis was undertaken.

\section{Mutational analysis}

The exonic regions from a panel of 218 IRD genes were sequenced by NGS in the proband from this pedigree. An additional 5 affected males were sequenced with a subsequent panel of the exonic regions of 254 IRD genes. Analysis of this NGS data led to the identification of a mutation in the RS1 gene within the pedigree. Further analysis of each of the members of the family by PCR amplification of RS1 and Sanger sequencing demonstrated that each affected male was found to have a hemizygous nonsynonymous mutation in exon 5 of the RS1 gene (NM_000330.3:c.413C > A, p.Thr138Asn). In addition, the single carrier female analyzed was heterozygous for the same $R S 1$ mutation while the unaffected male was hemizygous for the reference base at that position (Fig. 4a, b).

The p.Thr138Asn RS1 mutation involves a polar to polar amino acid substitution. Given that this novel as yet previously unreported mutation is located in the critical discoidin domain of the retinoschisin protein, where many pathogenic mutations have been identified previously, additional in silico analysis was undertaken to explore the potential pathogenicity of this RS1 mutation on protein structure and conformation. The destabilizing effect of this mutation as predicted in silico is shown below in terms of a prediction of the changes to fold stability (Table 3), where a negative delta delta $\mathrm{G}$ (ddG) score is indicative of a destabilizing effect. This change is also represented visually as theoretical structural changes to the retinoschisin monomeric protein structure (Fig. 5a, b). Notable secondary structural changes are identified with arrows in Fig. 5a while Fig. 5b clearly visualizes the position of the specific amino acid substitution. The complexity of the hexadecameric structure of retinoschisin is shown in Fig. 6 for reference.

Table 1 displays the comparative output scores of three highly regarded variant pathogenicity prediction tools for this novel variant and a known pathogenic variant. As seen in the respective columns of the tools, both variants have been deemed likely pathogenic by all three tools. Also, the novel variant described in this study is scored more likely to be pathogenic by two out of three methods listed.

The ACMG guidelines [31] have also been implemented to classify the novel variant. The relevant information has been outlined in Table 4 along with supporting evidence where appropriate [20,36, 37]. The guidelines state that there is sufficient evidence to classify this variant as pathogenic as there are one strong (PS1) and three moderate (PM1, PM2 and PM5) lines of evidence.

\section{Discussion}

A key function of retinoschisin is in retinal adhesion [7]. There is some debate over which retinal plane is affected by the schisis, which has been documented on OCT at the level of the photoreceptor inner segments, the inner nuclear layer and the nerve fibre layer [13, 38]. Decreased retinoschisin protein function may, and likely does, cause multiple planes of schisis.

There is significant variability of the severity of disease phenotype between the two eyes of the same individual, between different members of this XLRS1 pedigree and, as previously documented, between unrelated individuals [10-12]. This variability appears greater with age with some patients progressing through the clinical stages at different rates, possibly due to acquired/environmental factors or other genetic modifiers (see below). Although this variability is noted, there are clinical characteristics that guide the clinician to this diagnosis (e.g. pedigree, 


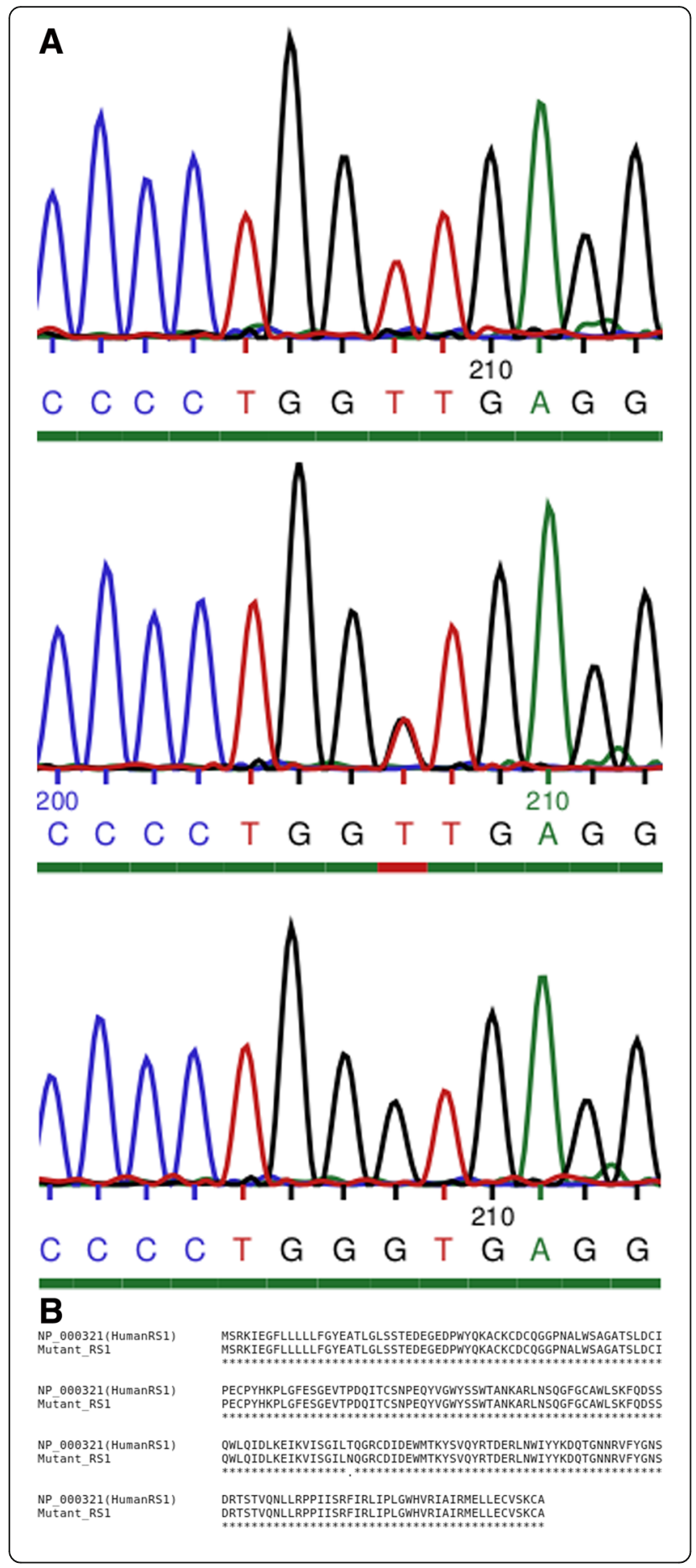

Fig. 4 a Sanger sequencing of RS1-exon 5 polymerase chain reaction $(P C R)$ products from members of the pedigree. Top: Sanger sequencing result for affected male (ID\#1392) showing a T nucleotide trace at sequencing position 208. Middle: Sanger sequencing result for carrier female (ID\#1534) showing a heterozygous result at sequencing position 207, revealing traces from both signals of wild-type $\mathrm{G}$ allele and the mutant $\mathrm{T}$ allele. Bottom: Sanger sequencing result for unaffected male (ID\#1634) showing a $\mathrm{G}$ nucleotide trace at sequencing trace position 208. b Clustal Omega results for protein alignment of reference RS1 protein sequence against the observed mutant protein sequence. "." denotes the position of the substitution

peripheral retinoschisis, lack of subretinal flecks or vitelliform changes) which are supported by a likely-pathogenic $R S 1$ variant and lack of other known pathogenic genetic variants. This supports the bimodal presentation of XLRS1. Severe cases of the disease (approximately 30\%) are detected in infancy with poor fixation and/or strabismus. Milder cases (approximately $70 \%)$ are detected at school entry or in adulthood [39]. The current study highlights that the underlying genetic variant on the RS1 gene is not predictive of phenotypic severity for this, or indeed any of the other, genetic variant(s) that have been described [12]. Thus, in assessing patients for trial/intervention suitability both a genetic and full phenotypic analysis is required. For example, gene therapy will not be beneficial if there is significant structural change (i.e. stage 3). However, the combination of a stage 1 or 2 case with confirmed genotype would suggest a possible role for genetic manipulation/ replacement. As our knowledge of the impact of multiple variants increases we may better predict the molecular effects of each on retinal structure and function thus tailoring our approach to intervention further.

The clinical findings may be subtle in early stage or mild forms of disease. Classically, radial cystic maculopathy at the fovea is seen in 98-100\% of cases [40] (Fig 2a-c). Ancillary tests have proven useful, particularly in subtle cases. An electronegative electroretinogram is suggestive of, but not specific, to XLRS1 [41] as this feature may be seen in acquired disease of the inner retina (e.g. central retinal artery occlusion). Microperimetry may assist in detection of subclinical disease [15]. Late stage disease is a diagnostic challenge; however, clinical examination, family history and genotyping as outlined above will aid in determining the diagnosis.

Colour photography, Autofluorescence and Optical Coherence Tomography are non-invasive and accessible

Table 3 Output results of the STRUM program. The change in stability scores between the mutant and wild type RS1 protein is given as delta delta $\mathrm{G}(\mathrm{ddG})$

\begin{tabular}{llll}
\hline Position & Wild Type & Mutant Type & ddG \\
\hline 138 & $\mathrm{~T}$ & $\mathrm{~N}$ & -0.78 \\
\hline
\end{tabular}



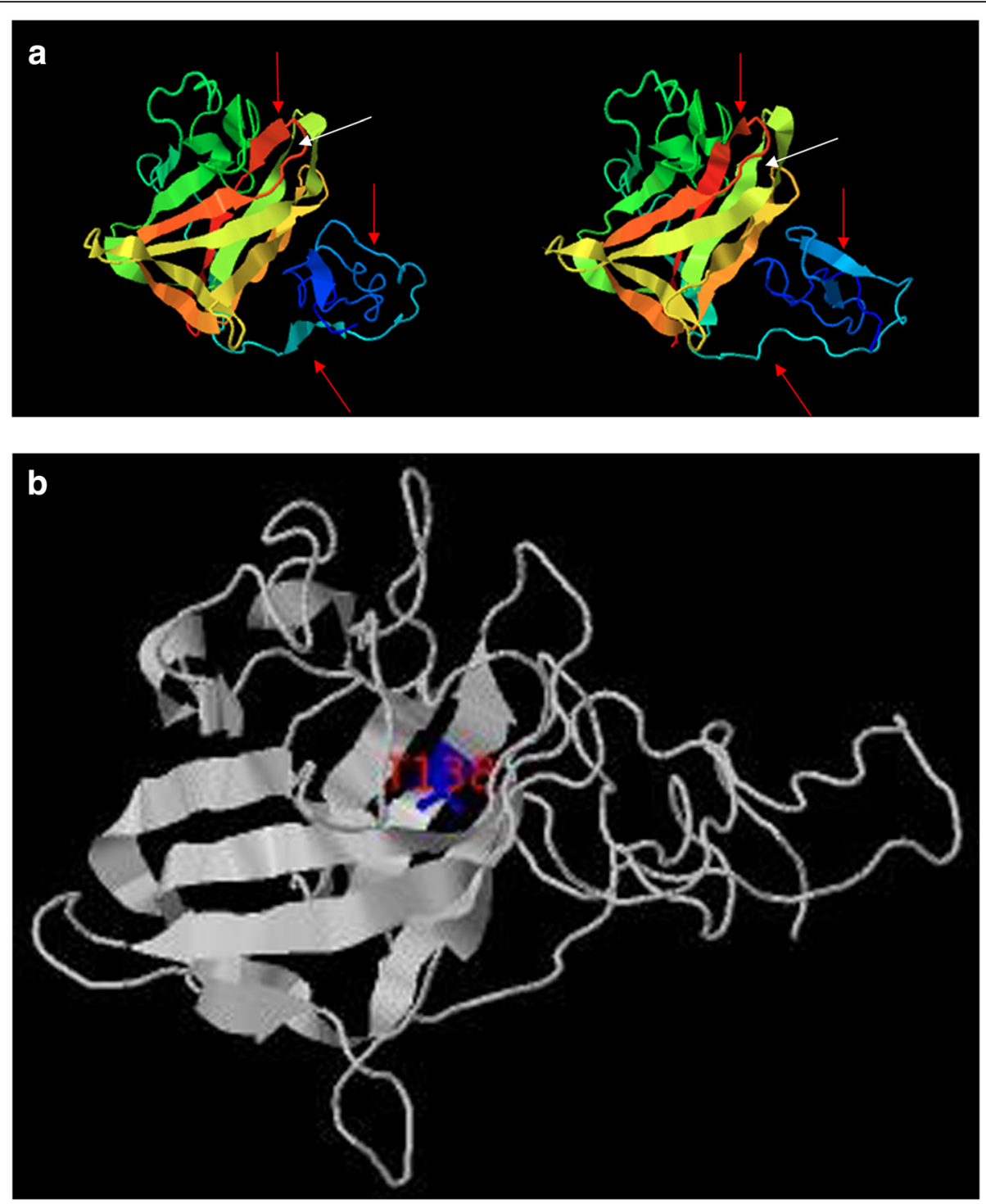

Fig. 5 a Protein models of reference RS1 (left) and mutant T138 N (right) proteins generated by the I-TASSER program. Red arrows denote obvious structural changes to protein structures. A white arrow highlights the position of the amino acid change at codon 138. $\mathbf{b}$ Protein model of RS1 mutant T138 N generated by STRUM. The affected amino acid residue is displayed in blue and annotated in red
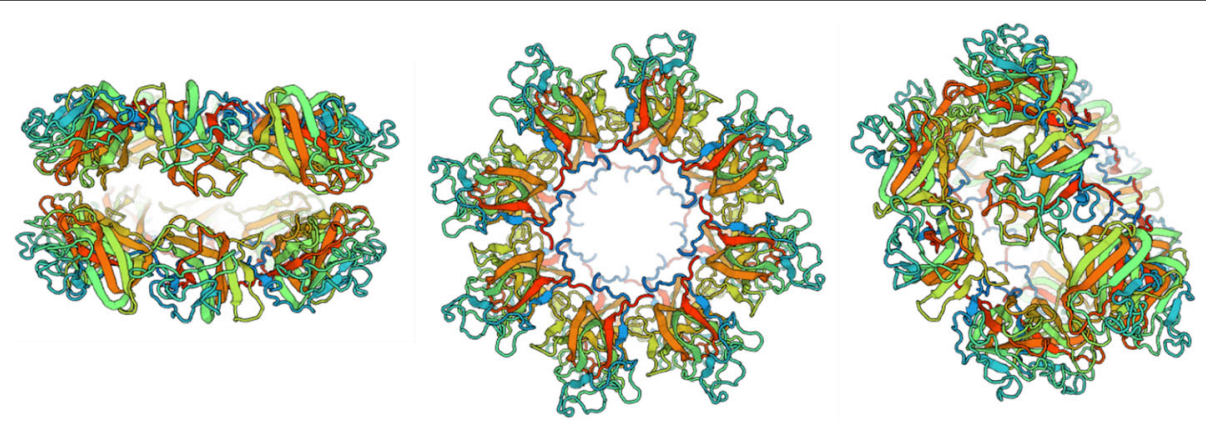

Fig. 6 Different views of the back-to-back octamer rings of wild type RS1 obtained from the Protein Data Bank. (Left) Objective view. (Middle) Plan view. (Right) Perspective view 
Table 4 The ACMG guidelines applicable to the novel variant in question, p. Thr128Asn

\begin{tabular}{|c|c|c|}
\hline Category Code & Qualifying Criteria & Relevance to Case \\
\hline PS4 & $\begin{array}{l}\text { The prevalence of the variant in affected individuals is } \\
\text { significantly increased compared to the prevalence in controls. }\end{array}$ & $\begin{array}{l}\text { This variant is found in a sufficient number of relatives } \\
\text { to qualify it as strong evidence of pathogenicity [36]. }\end{array}$ \\
\hline PM1 & $\begin{array}{l}\text { Located in a mutational hot spot and/or critical and well-established } \\
\text { functional domain (e.g. active site of an enzyme) without benign } \\
\text { variation. }\end{array}$ & $\begin{array}{l}\text { Located in well established functional domain } \\
\text { as reported by several studies }[20,37] \text {. }\end{array}$ \\
\hline PM2 & $\begin{array}{l}\text { Absent from controls (or at extremely low frequency if recessive) } \\
\text { in Exome Sequencing Project, } 1000 \text { Genomes or ExAC. }\end{array}$ & Absent from every databased queried. \\
\hline PM5 & $\begin{array}{l}\text { Novel missense change at an amino acid residue where a different } \\
\text { missense change determined to be pathogenic has been seen before. }\end{array}$ & $\begin{array}{l}\text { Novel mutation at same amino acid as previously } \\
\text { reported p.Thr138Ala (HGMD Database). }\end{array}$ \\
\hline PP1 & $\begin{array}{l}\text { Co-segregation with disease in multiple affected family members } \\
\text { in a gene definitively known to cause the disease. }\end{array}$ & $\begin{array}{l}\text { Cosegregation in a family in a gene known to } \\
\text { cause disease. }\end{array}$ \\
\hline PP3 & $\begin{array}{l}\text { Multiple lines of computational evidence support a deleterious } \\
\text { effect on the gene or gene product. }\end{array}$ & $\begin{array}{l}\text { Multiple lines of computational evidence } \\
\text { presented here (Table 1). }\end{array}$ \\
\hline PP4 & $\begin{array}{l}\text { Patient's phenotype or family history is highly specific for a disease } \\
\text { with a single genetic etiology. }\end{array}$ & Patient phenotype is highly specific for a disease. \\
\hline
\end{tabular}

tests useful in determining the clinical stage of XLRS1. This is relevant, for current and upcoming gene therapy trials $[8,9]$, in selecting those cases most likely to benefit from intervention (i.e. early to intermediate disease [40]). Ancillary tests, such as adaptive optics (AO) imaging and microperimetry, can complement assessment of cone structure/spacing in those who are deemed early/intermediate stage $[42,43]$.

To our knowledge, this specific mutation is currently unreported in any clinical database available including the Leiden University's Open Variation Database for Retinoschisis (LOVD 3.0 [6]) and represents a novel and likely pathogenic mutation given the clear segregation in this pedigree and the significant predicted effects on protein structure.

Recent studies have outlined the importance of certain key residues in correctly assembling a functional RS1 oligomer. Several key residue positions necessary to ensure proper folding of the retinoschisin protein have been identified [20]. Of note, amino acids at positions 137 and 139 were highlighted in that study as fundamental to forming a beta-sheet required in the interaction between subunits essential in the formation of their functional octamer ring structure. It is possible therefore, that an amino acid substitution at position 138 may provide some steric hindrance, that in turn, would interfere with the relationship between different subunits on the same octamer ring. Such interference could in principle have deleterious consequences for the formation and stability of the oligomeric RS1 complex. This hypothesis is further supported by in silico analysis of the p.Thr138Asn mutation. Computational analysis was utilized to predict protein folding changes in the mutant protein (using I-TASSER); physiochemical properties, position specific conservation and secondary structure prediction scores were used to determine the destabilization effect(s) of the protein substitution at position 138 (using STRUM) where significant changes in the above were predicted for the p.Thr138Asn mutation.

The prediction scores in Table 1 are useful for assessing the confidence with which a variant can be called deleterious or not. However, these are confidence scores and not pathogenicity scores, so although the scores listed would be considered to be quite high, these scores cannot indicate the detrimental effect that this mutation may have relative to a similar amino acid substitution elsewhere in the protein. Increased bioavailability of functional retinoschisin protein would logically correlate to the lasting preservation of the retinal layers; however, to assess this dosage effect, each variant would have to be assessed individually for function and half-life. This is a challenging task for retinoschisin as it has typically proven quite difficult to produce in useful quantities [20]. Currently we rely on in silico predictors of a variant's effect on protein structure. This includes protein modelling (Figs. 4a and b) where possible and delta delta g scores (Table 3), to predict the destabilising effects a mutation may have on a protein. However, when all lines of evidence are considered, this novel RS1 variant is classified as pathogenic under the guidelines outlined by the ACMG [31] (Table 4).

In XLRS1, age is not an absolute factor, particularly in adulthood, when evaluating severity of disease and the likely benefit from novel gene therapies. The individuals in this XLRS1 pedigree exhibited no clear correlation between patient age and 1) stage of disease, 2) visual acuity, 3) central retinal thickness or 4) age at onset despite a shared novel $R S 1$ gene variant. Advanced cases prove a diagnostic challenge as many inherited and acquired macular diseases follow a final common pathway of outer retinal atrophy. Each case must be judged on its potential (anatomically within Stage 1 or 2 and 
genetically possessing a pathogenic $R S 1$ variant) to respond to novel therapies. Furthermore, the variability in clinical presentations between family members with the same mutation suggests the possible involvement of genetic modifier loci and or environmental effect(s).

While there is variability in phenotype between relatives affected by the same genetic variant, the significance is that the clinician not be discouraged from the diagnosis due to these differences but consider them within the spectrum of the 3 described clinical stages. The diagnostic value of ERG, in particular the characteristic decrease in b-wave activity in combination with suggestive clinical findings are powerful tools for diagnosing younger patients, however the diagnostic value of these tests may be less certain with advanced age [44]. Confirmation of extent of severity (clinical stages) and a pathogenic $R S 1$ variant open a potential avenue of treatment for those in Stage 1 or 2. This information then guides genetic testing for at risk relatives who could possibility benefit from novel disease-modifying therapies.

There is a possibility that there may be some form of modifier or digenic effect which could account for the observed phenotypic variability. With this in mind, the coding regions of known genes associated with retinal degenerations were subjected to sequence analysis. The presence of multiple pathogenic variants within a single patient is extremely rare but has been previously observed, even in our own IRD cohort. Although, as previously mentioned, this method unfortunately did not detect any likely genetic cause of phenotypic variability between the affected patients in this pedigree.

The retinoschisin protein is widely expressed throughout the layers of the retina in childhood and more specifically in rod and cone photoreceptor cells in adulthood and is seen to act as a cellular adhesive when functioning correctly. However, other proteins are also capable of fulfilling similar adhesive functions, for example adherins and cadherins in adherens junctions or claudins and occuldin in tight junctions. Given that some of these other proteins belong to large protein families, it is a possibility that variation in relative composition of these cellular adhesive protein cocktails may have a more notable contribution to a variable phenotype in the absence of functional retinoschisin.

This variability creates a diagnostic challenge in advanced XLRS1 disease. The clinical clues in conjunction with adjunctive testing, confirmed by robust genetic assessment (i.e. ruling out other causes and confirming a pathogenic RS1 variant) confirm the diagnosis, thus empowering those affected by bringing them closer to accessing novel gene therapies where appropriate and preparing younger generations for those potential therapeutic options.

\section{Conclusions}

Here we describe how multimodal imaging in XLRS1 and confirmation of a pathogenic $R S 1$ variant help to a) confirm diagnosis, b) monitor progression, c) assess clinical stage and thus suitability for novel therapeutic options even in patients of advanced age. Autofluorescence and OCT are non-invasive, well-tolerated and readily available in most clinical centres. Standardisation of clinical assessment and staging criteria, perhaps in conjunction with a central European reading centre, would allow detection of individuals with modifiable XLRS1 who may be suitable for novel gene therapy clinical trial participation.

\section{Abbreviations}

ACMG: American College of Medical Genetics; AF: Autofluorescence; AO: Adaptive Optics; IRD: Inherited Retinal Degeneration; I-TASSER: Iterative

Threading ASSEmbly Refinement; LogMAR: Logarithm of the Minimum Angle of Resolution; LOVD: Leiden University's Open Variation Database;

MMUH: Mater Misericordiae University Hospital, Dublin, Ireland; MPH: Mater Private Hospital, Dublin, Ireland; OCT: Optical Coherence Tomography; OMIM: Online Mendelian Inheritance in Man; PCR: Polymerase Chain Reaction; PDB: Protein Data Bank; RPE: Retinal Pigment Epithelium; RVEEH: Royal Victoria Eye \& Ear Hospital, Dublin, Ireland; XLRS1: X-Linked Retinoschisis

\section{Acknowledgements}

Fighting Blindness Ireland; Clinical Research Centre: University College Dublin/Mater Misericordiae University Hospital, Dublin, Ireland; The Research Foundation, The Royal Victoria Eye \& Ear Hospital, Dublin, Ireland; School of Genetics, Trinity College Dublin, Ireland; Retinal Eye Centre, Mater Private Hospital, Dublin, Ireland.

\section{Funding}

All patients were seen as part of the Target 5000 Irish National Inherited Retinal Degeneration Registry which is funded through the Irish charity Fighting Blindness (charity number: 20013349). There are no specific sources of industry or institutional funding supporting this publication. All publications arising as part of this project are independent endeavours of the publishing authors.

\section{Availability of data and materials}

All source notes are securely stored at the Catherine McAuley Research \& Education Centre, Nelson Street, Dublin 7 or the Research Foundation, Royal Victoria Eye \& Ear Hospital, Adelaide Road, Dublin 2. The 2 children were recruited via RVEEH while all adults were recruited via MMUH/MPH. Source documentation is available upon request from the corresponding author.

\section{Authors' contributions}

KS, NW, PK and DK recruited the patients, performed history and clinical examination, and wrote the clinical portions of the manuscript. AD, MC and GJF performed next generation sequencing on blood samples from the patients, performing protein modelling/pathogenicity analysis and wrote the genetic portions of the manuscript. All authors have read and approved the manuscript.

Ethics approval and consent to participate

This study was approved by the Institutional Review Boards of the Mater Misericordiae University Hospital, Mater Private Hospital and Royal Victoria Eye \& Ear Hospital, Dublin, Ireland. All patients (or their legal guardians in the case of children) whose data is described in this manuscript signed informed consent before taking part in this study. This study is in accordance with the Declaration of Helsinki.

\section{Consent for publication}

All patients (or their legal guardians in the case of children) whose data is described in this manuscript signed informed consent giving permission for their data to be published anonymously. 


\section{Competing interests}

None of the authors have any competing interests as laid out in BioMed Central's guidance document.

\section{Publisher's Note}

Springer Nature remains neutral with regard to jurisdictional claims in published maps and institutional affiliations.

\section{Author details}

${ }^{1}$ The Catherine McAuley Centre, Mater Private Hospital, Nelson Street, Dublin 7, Ireland. ${ }^{2}$ Department of Genetics, Trinity College Dublin, Dublin, Ireland. ${ }^{3}$ The Research Foundation, The Royal Victoria Eye and Ear Hospital, Dublin, Ireland.

Received: 4 October 2017 Accepted: 29 October 2018

Published online: 12 November 2018

\section{References}

1. Carrigan $\mathrm{M}$, et al. Panel-based population next-generation sequencing for inherited retinal degenerations. Sci Rep. 2016;6:33248.

2. Dockery A, et al. Target 5000: Target Capture Sequencing for Inherited Retinal Degenerations. Genes (Basel). 2017;8(11). https://doi.org/10.3390/ genes8110304.

3. George ND, et al. Infantile presentation of $X$ linked retinoschisis. $\mathrm{Br} J$ Ophthalmol. 1995;79(7):653-7.

4. Functional implications of the spectrum of mutations found in 234 cases with X-linked juvenile retinoschisis. The Retinoschisis consortium. Hum Mo Genet. 1998;7(7):1185-92. https://www.ncbi.n/m.nih.gov/pubmed/9618178.

5. Stenson PD, et al. The human gene mutation database: building a comprehensive mutation repository for clinical and molecular genetics, diagnostic testing and personalized genomic medicine. Hum Genet. 2014; 133(1):1-9.

6. Leiden University Medical Center, N. Leiden Open Variation Database 3.0. 2018; Available from: http://www.lovd.nl/3.0/home. Accessed 5 July 2018.

7. Bush M, et al. Cog-wheel Octameric structure of RS1, the Discoidin domain containing retinal protein associated with X-linked Retinoschisis. PLoS One. 2016;11(1):e0147653.

8. ClinicalTrials.gov [Internet]. Bethesda (MD): National Library of Medicine (US). 2015 Apr 15. Identifier NCT02416622, Safety and Efficacy of rAAV-hRS1 in Patients With X-linked. Retinoschisis (XLRS); [6 screens]. Available from: https:/clinicaltrials.gov/ct2/show/NCT02416622. Accessed 5 July 2018.

9. ClinicalTrials.gov [Internet]. Bethesda (MD): National Library of Medicine (US) 2014 Dec 14. Identifier NCT02317887, Study of RS1 Ocular Gene Transfer for X-linked Retinoschisis; [8 screens]. Available from: https://clinicaltrials.gov/ ct2/show/NCT02317887. Accessed 5 July 2018.

10. Eksandh LC, et al. Phenotypic expression of juvenile X-linked retinoschisis in Swedish families with different mutations in the XLRS1 gene. Arch Ophthalmol. 2000;118(8):1098-104.

11. Molday RS, Kellner U, Weber BH. X-linked juvenile retinoschisis: clinical diagnosis, genetic analysis, and molecular mechanisms. Prog Retin Eye Res. 2012;31(3):195-212.

12. Lai $\mathrm{YH}$, et al. A novel gene mutation in a family with $\mathrm{X}$-linked retinoschisis. J Formos Med Assoc. 2015;114(9):872-80.

13. Gregori NZ, et al. Macular spectral-domain optical coherence tomography in patients with X linked retinoschisis. Br J Ophthalmol. 2009;93(3):373-8.

14. Tsui I, Tsang SH. In: Noemi Lois JVF, editor. Fundus autofluorescence in X-linked Retinoschisis, in Fundus Autofluorescence. Philadelphia: Lippincott Williams \& Wilkins; 2009.

15. Nittala MG, et al. Spectral-domain OCT and microperimeter characterization of morphological and functional changes in X-linked retinoschisis. Ophthalmic Surg Lasers Imaging. 2009;40(1):71-4.

16. Bergen $A A$, et al. Multipoint linkage analysis in $X$-linked juvenile retinoschisis. Clin Genet. 1993;43(3):113-6.

17. Sauer CG, et al. Positional cloning of the gene associated with X-linked juvenile retinoschisis. Nat Genet. 1997;17(2):164-70.

18. Plossl $K$, et al. Retinoschisin is linked to retinal Na/K-ATPase signaling and localization. Mol Biol Cell. 2017;28(16):2178-89.

19. Plossl $K$, Weber $B H$, Friedrich $U$. The $X$-linked juvenile retinoschisis protein retinoschisin is a novel regulator of mitogen-activated protein kinase signalling and apoptosis in the retina. J Cell Mol Med. 2017;21(4):768-80.
20. Tolun $\mathrm{G}$, et al. Paired octamer rings of retinoschisin suggest a junctional model for cell-cell adhesion in the retina. Proc Natl Acad Sci U S A. 2016; 113(19):5287-92.

21. Ramsay EP, et al. Structural analysis of X-linked retinoschisis mutations reveals distinct classes which differentially effect retinoschisin function. Hum Mol Genet. 2016;25(24):5311-20.

22. den Hollander Al, et al. Mutations in the CEP290 (NPHP6) gene are a frequent cause of Leber congenital amaurosis. Am J Hum Genet. 2006;79(3): 556-61.

23. Bauwens $M$, et al. An augmented ABCA4 screen targeting noncoding regions reveals a deep intronic founder variant in Belgian Stargardt patients. Hum Mutat. 2015;36(1):39-42.

24. Steele-Stallard HB, et al. Screening for duplications, deletions and a common intronic mutation detects $35 \%$ of second mutations in patients with USH2A monoallelic mutations on sanger sequencing. Orphanet J Rare Dis. 2013;8:122.

25. Li H, Durbin R. Fast and accurate long-read alignment with burrowswheeler transform. Bioinformatics. 2010;26(5):589-95.

26. Picard. Available from: http://broadinstitute.github.io/picard/. Accessed 18 Aug 2017.

27. Garrison E, Marth G. Haplotype-based variant detection from short-read sequencing. arXiv:1207.3907 2012; Available from: http://arxiv.org/abs/1207.3907.

28. Dong C, et al. Comparison and integration of deleteriousness prediction methods for nonsynonymous SNVs in whole exome sequencing studies. Hum Mol Genet. 2015;24(8):2125-37.

29. Jagadeesh KA, et al. M-CAP eliminates a majority of variants of uncertain significance in clinical exomes at high sensitivity. Nat Genet. 2016;48(12): 1581-6.

30. loannidis NM, et al. REVEL: an ensemble method for predicting the pathogenicity of rare missense variants. Am J Hum Genet. 2016;99(4):877-85.

31. Richards S, et al. Standards and guidelines for the interpretation of sequence variants: a joint consensus recommendation of the American College of Medical Genetics and Genomics and the Association for Molecular Pathology. Genet Med. 2015;17(5):405-24.

32. I-TASSER. Available from: http://zhanglab.ccmb.med.umich.edu/l-TASSER/. Accessed 18 Aug 2017.

33. RCSB Protein Data Bank. Available from: https://www.rcsb.org/. Accessed 18 Aug 2017.

34. Quan L, LV Q, Zhang Y. STRUM: structure-based prediction of protein stability changes upon single-point mutation. Bioinformatics. 2016;32(19): 2936-46.

35. Sievers F, et al. Fast, scalable generation of high-quality protein multiple sequence alignments using Clustal omega. Mol Syst Biol. 2011;7:539.

36. Jarvik GP, Browning BL. Consideration of Cosegregation in the pathogenicity classification of genomic variants. Am J Hum Genet. 2016; 98(6):1077-81.

37. Ploss $K$, et al. Pathomechanism of mutated and secreted retinoschisin in X-linked juvenile retinoschisis. Exp Eye Res. 2018;177:23-34.

38. Yanoff M, Kertesz Rahn E, Zimmerman LE. Histopathology of juvenile retinoschisis. Arch Ophthalmol. 1968;79(1):49-53.

39. Deutman AF, Pinckers AJ, Aan de Kerk AL. Dominantly inherited cystoid macular edema. Am J Ophthalmol. 1976;82(4):540-8.

40. Wang $T$, et al. Intracellular retention of mutant retinoschisin is the pathological mechanism underlying X-linked retinoschisis. Hum Mol Genet. 2002:11(24):3097-105.

41. Sergeev $\mathrm{Y}$, et al. Molecular modeling of retinoschisin with functional analysis of pathogenic mutations from human X-linked retinoschisis. Hum Mol Genet. 2010;19(7):1302-13.

42. Akeo $\mathrm{K}$, et al. Detailed morphological changes of Foveoschisis in patient with X-linked Retinoschisis detected by SD-OCT and adaptive optics fundus camera. Case Rep Ophthalmol Med. 2015;2015:432782.

43. Duncan $J$, et al. Abnormal cone structure in foveal schisis cavities in $X$-linked retinoschisis from mutations in exon 6 of the RS1 gene. Invest Ophthalmol Vis Sci. 2011:52(13):9614-23.

44. Bradshaw K, et al. Mutations of the XLRS1 gene cause abnormalities of photoreceptor as well as inner retinal responses of the ERG. Doc Ophthalmol. 1999;98(2):153-73.

45. McCulloch DL, et al. ISCEV standard for full-field clinical electroretinography (2015 update). Doc Ophthalmol. 2015;130(1):1-12. 